\title{
Ground State Energies of a Sextic Anharmonic Oscillator Including Quartic Anharmonicity
}

\author{
Gayathri G. M. ${ }^{1}$, B. A. Kagali ${ }^{2}$, T. Shivalingaswamy ${ }^{3}$ \\ ${ }^{1}$ Department of Physics, HKES (SVP) College, Bangalore-560081, India \\ ${ }^{2}$ Department of Physics, Bangalore University, Bangalore-560056, India \\ ${ }^{3}$ Department of Physics, Government College (Autonomous), Mandya-571401, India
}

\begin{abstract}
The ground state energy eigenvalues of a sextic anharmonic oscillator including quartic anharmonicity are obtained using the Ginsberg-Montroll method. Numerical values are calculated using Mathematica for different values of the anharmonic coefficients.
\end{abstract}

Keywords: Quantum anharmonic oscillators; Ginsberg-Montroll method; Approximation Methods; Energy eigenvalues; Mathematica

PACS: 03.65.Ge; 02.30.Gp; 02.30.Hq

\section{Introduction}

Quantum mechanical anhormonic oscillators (AHO) have been studied over the years due to their occurance in various fields of physics such as Field theories, Molecular Physics, Solid state physics etc. They have been studied by several methods both from analytical and the numerical points of view. For instance, they have been studied using WKB approximation [1, 2], Hill determinant method [3], action-angle technique [4], Continued fraction method [5], the variational method [6,7], Re-normalised frequency method [8] Chebyshev polynomial method [9], the residue-squaring method [10], Pade approximants method [11], the kinetic potential method [12], the fixed point method [13], the hypervirial method [14] and so on. In this paper we make use of one of the simplest methods proposed by Ginsberg and Montroll [15]. Using their new method Ginsberg and Montroll have determined the eigen energy values of a pure quartic oscillator. More recently the method has been applied by Shivalingaswamy and Kagali [16] for a pure sextic oscillator. However, on physical grounds we expect a quartic anharmonic term along with a sextic term for every anharmonic osillator. Thus we study an anharmonic oscillator having both quartic and sextic anharmonic terms.

\section{Ginsberg-Montroll Method}

In this method a wave function with proper asymptotic behavior is chosen. The wave function is then constructed with other interpolative terms. The coeficients of the interpolative powers are determined by solving the Schrodinger equation near the origin. The coeficients lead to a polynomial equation for the energy eigenvalues that results from the requirements of self consistency [15].

\section{The Sextic oscillator with quartic anharmonic term}

The time-dependent Schrödinger equation for a one dimensional oscillator with both sextic and quartic anharmonic potentials can be written as $\left\{-\frac{\hbar^{2}}{2 m} \frac{d^{2}}{d x^{2}}+\frac{1}{2} K x^{2}+\mu^{\prime} x^{4}+\lambda^{\prime} x^{6}-E\right\} \psi(x)=0$

By putting

$$
\omega^{2}=\frac{K}{m}, \lambda=\frac{\lambda^{\prime} \hbar^{2}}{m^{3} \omega^{3}}, \mu=\frac{2 \mu^{\prime} \hbar}{m^{2} \omega^{3}}, \varepsilon=\frac{E}{\hbar \omega}
$$

and changing the variable to

$$
y=\left(\frac{m \omega}{\hbar}\right)^{\frac{1}{2}} x
$$

we get the dimensionless equation:

$$
\left(\frac{1}{2} \frac{d^{2}}{d y^{2}}-\frac{1}{2} y^{2}-\mu y^{4}-\lambda y^{6}+\varepsilon\right) \psi(y)=0
$$

For large $|y|$,

$$
\psi(y) ; \exp \left(-\sqrt{\frac{\lambda}{8}} y^{4}\right)
$$

For small $|y|$,

$$
\psi(y) ; \exp \left(-\beta y^{2}\right)
$$

where $\beta$ is a numerical parameter.

Following Ginsberg and Montroll [15], we postulate the following interpolative wave function for the ground state

$$
\psi_{0}(y)=\text { const } \exp \left\{-\left(a_{1} y^{4}+a_{2} y^{6}+a_{3} y^{8}\right)^{\frac{1}{2}}\right\}
$$

We attempt to solve equation (2) near the origin of $y$. 


\section{International Journal of Science and Research (IJSR) \\ ISSN (Online): 2319-7064}

Index Copernicus Value (2013): 6.14 | Impact Factor (2015): 6.391

Hence expanding the exponent of the wave function $\psi_{0}$ in the powers of $y$ we get

$$
\psi_{0}(y)=\text { constexp }\left\{-\left(c_{2} y^{2}+c_{4} y^{4}+c_{6} y^{6}+\ldots \ldots \ldots .\right)\right\}
$$

where

$$
c_{2}=a_{1}^{\frac{1}{2}}, c_{4}=\frac{1}{2} a_{2} a_{1}^{-\frac{1}{2}}, c_{6}=\frac{\lambda}{16} a_{1}^{-\frac{1}{2}}-\frac{1}{8} a_{2}^{2} a_{1}^{-\frac{3}{2}}, \text { etc. }
$$

Therefore, near the origin we get

$$
\frac{1}{2} \frac{d^{2}}{d y^{2}} \psi_{0}(y) ;\left\{-c_{2}+2\left(c_{2}^{2}-3 c_{4}\right) y^{2}-\left(15 c_{6}-8 c_{2} c_{4}\right) y^{4}+\ldots \ldots \ldots . .\right\}
$$

Substituting equation (7) into equation (2) and equating the coefficients of $y^{0}, y^{2}, y^{4}$ to zero, we get the following equations:

The ground state Energy eigenvalues $\varepsilon\left(\frac{E}{\hbar \omega}\right)$ of a sextic anharmonic oscillator including quartic anharmonicity

$$
\begin{aligned}
& a_{1}^{\frac{1}{2}}-\varepsilon=0, \\
& -3 a_{2} a_{1}^{-\frac{1}{2}}+2 a_{1}-\frac{1}{2}=0 \\
& \text { and } 4 a_{2}-\frac{15}{2} a_{1}^{\frac{1}{2}}\left\{\frac{a_{3}}{a_{1}}-\frac{1}{4}\left(\frac{a_{2}}{a_{1}}\right)^{2}\right\}-\mu=0
\end{aligned}
$$

$a_{1}$ and $a_{2}$ can be solved in terms of $a_{3}$, where $a_{3}=\frac{\lambda}{8}$. Hence we get the following characteristic equation for $\varepsilon$ :

$$
1008 \varepsilon^{4}-312 \varepsilon^{2}-288 \mu \varepsilon-270 \lambda+15=0
$$

\section{The Energy eigenvalues}

Equation (9) can be solved using Mathematica [17] for different chosen values of the anharmonic coefficients $\mu$ and $\lambda$. The results are tabulated in Table 1 .

\begin{tabular}{|c|c|c|c|c|c|c|c|}
\hline$\lambda$ & $\mu=0$ & $\mu=0.1$ & $\mu=0.5$ & $\mu=1.0$ & $\mu=10$ & $\mu=100$ & $\mu=1000$ \\
\hline 0 & 0.500000 & 0.559512 & 0.695777 & 0.801717 & 1.49006 & 3.09068 & 6.60199 \\
\hline 0.01 & 0.512985 & 0.567431 & 0.699199 & 0.803799 & 1.49035 & 3.09072 & 6.60199 \\
\hline 0.1 & 0.586616 & 0.621538 & 0.727012 & 0.8215461 & 1.49288 & 3.09099 & 6.60202 \\
\hline 1.0 & 0.82528 & 0.835627 & 0.88996 & 0.945755 & 1.51736 & 3.09374 & 6.6023 \\
\hline 10 & 1.33946 & 1.34381 & 1.36104 & 1.3822 & 1.704 & 3.12067 & 6.60509 \\
\hline 100 & 2.30892 & 2.3103 & 2.31581 & 2.32268 & 2.44259 & 3.35012 & 6.6328 \\
\hline 1000 & 4.06465 & 4.06509 & 4.0683 & 4.06901 & 4.10805 & 4.47766 & 6.88755 \\
\hline 10000 & 7.20484 & 7.20498 & 7.20553 & 7.20622 & 7.21863 & 7.34153 & 8.45782 \\
\hline 20000 & 8.56431 & 8.5644 & 8.56479 & 8.56528 & 8.57406 & 8.66134 & 9.48539 \\
\hline
\end{tabular}

\section{Results and Discussions}

For $\lambda$ and $\mu$ both vanishing we clearly get the harmonic oscillator eigenvalue for $\varepsilon$, namely, 0.5 , as we should. As $\lambda$ and $\mu$ increase the ground state energies increase slowly. The method of evaluating the ground state energies is rather simple compared to other methods cited in the introduction and is very much valid for small values of $\mu$ and $\lambda$. By including more number of parameters in the interpolative wave function it may be possible to improve the numerical accuracy of the energy eigenvalues. Further, one can use this method to estimate the excited state energies of anharmonic oscillators by using a wave function that is a product of a suitable polynomial with the ground state wave function used in this article.

\section{Acknowledgements}

The authors thank Dr. Sathyanarayana, Department of mathematics, Vijaya College, Bangalore for his assistance in computations using Mathematica.

\section{References}

[1] E. Merzbacher, Quantum Mechanics, (India: John Wiley and Sons) (Third Edition), pp 123, (2004).

[2] M. Seetharaman, Sekhar Raghavan and S.S Vasan, Analytic WKB energy expressions for three dimensional anharmonic oscillators, J. Phys. A, 15(5), pp 1537, (1982).

[3] S.N Biswas, K. Dutta, R.P Saxena, P.K Srivastava and V.S Varma, Hill determinant:An application to the anharmonic oscillator, Phys. Rev. D, 4(12), pp 3617, (1971).

[4] Gin-Yih Tsaur and Jyhpyng wang, Chinese J.Phys. 49(2), pp555, (2011).

[5] R.N Choudhuri, Phys. Rev. D, 31(10), pp2687, (1985).

[6] W. N Mei, Combined variational-perturbative approach to anharmonic oscillator problems, Int. J. Math. Edu. Sci. Tech, 29(6), pp 875, (1998).

[7] V.A Popescu, A new form of the successive variational method for the D-dimensional generalized anharmonic oscillator, Phy. Lett. A, 193, pp 431, (1994).

[8] P.M Mathews, M. Seetharaman, Sekhar Raghavan and V.T. Bhargava, A simple accurate formula for the 


\section{International Journal of Science and Research (IJSR) \\ ISSN (Online): 2319-7064}

Index Copernicus Value (2013): 6.14 | Impact Factor (2015): 6.391

energy levels of oscillators with a quartic potential, Phys. Lett. 83A(3), pp 118, (1981).

[9] J.P Boyd, J. Math. Phys., 19, pp 1445, (1978).

[10]P.M Mathews, Pramana, 4, pp 53, (1975).

[11] J. Leffel et.al., Phys. Lett. B, 30, pp 656, (1969).

[12] R. L Hall, Can.J. Phys. 63, pp 311, (1985).

[13]Znojil M and Tater, J. Phys. A, 21, pp 3217, (1986).

[14] Killingbech J., J.Phys. A, 20, pp 601, (1987).

[15]C.A Ginsberg and E.W Montroll, Application of a novel interpolative perturbation scheme to the determination of anharmonic wavefunctions, J. Math. Phys., 19(1), pp 336, (1978).

[16] T. Shivalingaswamy and B.A Kagali, Ground States of Sextic and Octic Anharmonic Oscillators, Paripex-Indian Journal of Research, 3(7), pp 49, (2014).

[17] Stephen Wolfram, The Mathematica Book and Software, (Cambridge:Cambridge University Press), (1996)

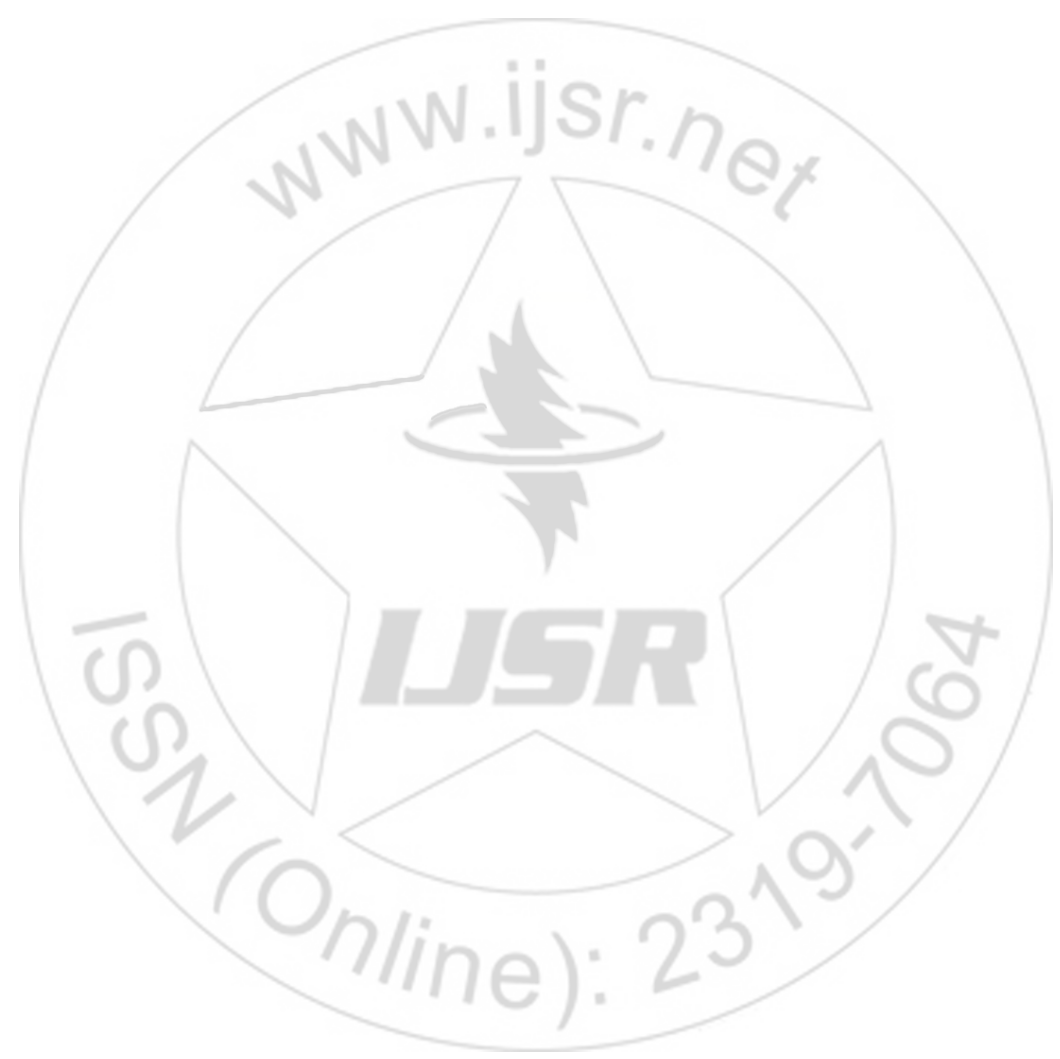

Volume 5 Issue 5, May 2016 